\title{
Universal and Nonuniversal Aspects of Electrostatics in Aqueous Nanoconfinement
}

\author{
Philip Loche, Cihan Ayaz, Amanuel Wolde-Kidan, Alexander Schlaich, and Roland R. Netz*
}

Cite This: J. Phys. Chem. B 2020, 124, 4365-4371

Read Online

ABSTRACT: Dielectric water properties, which significantly change in confinement, determine electrostatic interactions and thereby influence all molecular forces and chemical reactions. We present comparative simulations of water between graphene sheets, decanol monolayers, and phospholipid and glycolipid bilayers. Generally, dielectric profiles strongly differ in perpendicular and parallel surface directions and for large surface separation decay to the bulk value $1-2 \mathrm{~nm}$ away from the surface. Polar surface groups enhance the local interfacial dielectric response and for phospholipid bilayers induce a giant parallel contribution. A mapping on a box model with asymptotically determined effective water layer widths demonstrates that the perpendicular effective dielectric constant for all systems decreases for confinement below a nanometer, while the parallel one stays rather constant. The confinement-dependent perpendicular effective dielectric constant for graphene is in agreement with experimental data only if the effective water layer width is suitably adjusted. The interactions between two charges at small separation depend on the product of parallel and perpendicular effective water

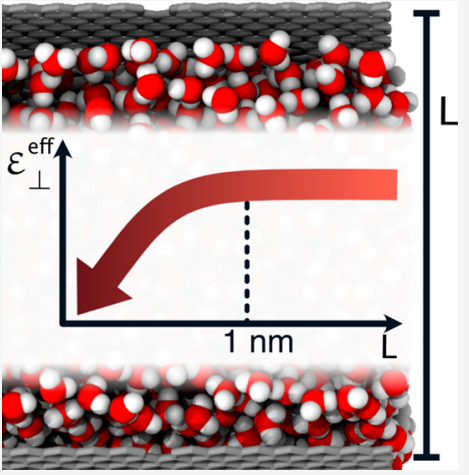
dielectric components; for large separation the interactions depend on the confining medium.

For metallic confining media the interactions at large separation decay exponentially with a decay length that depends on the ratio of the effective parallel and perpendicular water dielectric components.

\section{INTRODUCTION}

Biologically and technologically relevant surfaces in contact with water exhibit a wide range of elastic behavior from soft (e.g., lipid bilayers) to hard (e.g., minerals and metal oxide surfaces) and a wide range of polarities from surfaces that are completely wetted by water to surfaces that are hydrophobic, such as graphene. In the past decades nanometer cavities filled with water moved into the focus because of novel assembly methods and promising applications. ${ }^{1-10}$ It is generally acknowledged that changed water properties in nanopores and nanoslits significantly modify surface interactions, ionic adsorption, molecular transport, and chemical reaction equilibria, $^{11-21}$ but the precise mechanisms behind this relation are not clear. One fundamental property of water is its dielectric constant, which directly affects electrostatics and thereby influences, among other phenomena, surface interactions and electrokinetics. ${ }^{2-24}$ Clearly, all models and theories that involve charges need as input precise characterization of the electrostatic interactions, which in turn depend on the dielectric properties. By simulations and experiments, it was shown that for planar confined systems, the dielectric properties of water become anisotropic. ${ }^{24-31}$ In particular, it was recently demonstrated that the effective perpendicular dielectric component decreases significantly in strong confinement. ${ }^{29,30}$ This would suggest that ion-ion interactions in water change dramatically in nanoconfinement and that all static and kinetic electrolyte properties are modified as well. But how to derive meaningful effective dielectric quantities from simulations and experiments, how to calculate ion-ion interactions in confinement in the presence of tensorial dielectric constants, and the influence of the surface type on the water dielectric properties in confinement are far from settled. A fundamental problem that shows up in this context is that the effective tensorial dielectric constants of nanoconfined water depend on details of the model used for their definition. In particular, for a box model, where the effective dielectric constants are assumed to be constant in a slab of finite width, the effective dielectric constants depend sensitively on the chosen slab thickness, as has been demonstrated in experiments $^{30}$ and simulations. ${ }^{29,32}$ This problem can in fact be elegantly solved in molecular simulations, since the local dielectric constants in the water slab center can be directly accessed in a model-free fashion and used to check the validity of the model employed in the definition of the effective dielectric constants.

Received: March 5, 2020

Revised: April 27, 2020

Published: May 4, 2020 
(a)
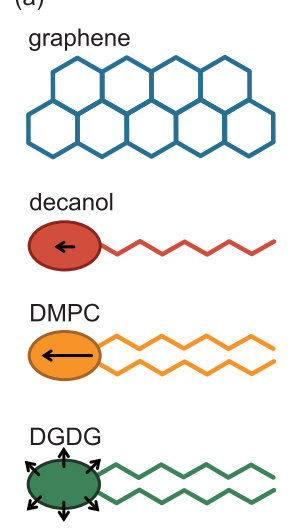
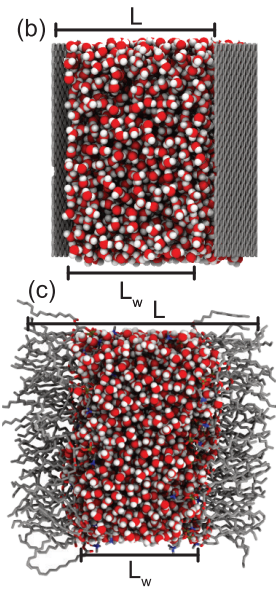

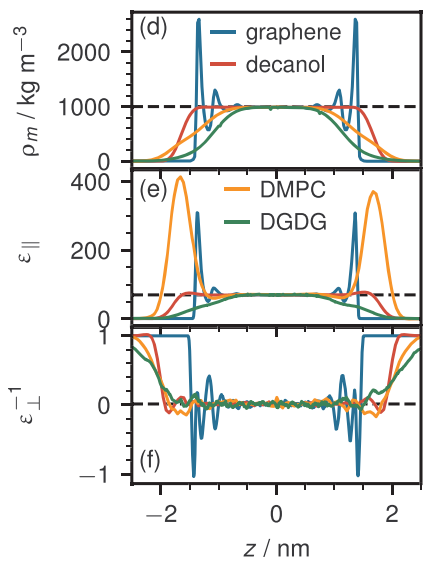

Figure 1. (a) Schematics of the constituents of the different surfaces. (b) Simulation snapshots of the graphene (water slab thickness $L_{\mathrm{w}}=3.0 \mathrm{~nm}$ ) and (c) the DMPC lipid bilayer system $\left(L_{\mathrm{w}}=2.9 \mathrm{~nm}\right)$. (d) Water mass density profiles $\rho_{\mathrm{m}}(z)$, (e) parallel dielectric profiles $\varepsilon_{\|}(z)$, and (f) inverse perpendicular dielectric profiles $\varepsilon_{\perp}^{-1}(z)$ for graphene $\left(L_{\mathrm{w}}=3.0 \mathrm{~nm}\right)$, decanol $\left(L_{\mathrm{w}}=3.3 \mathrm{~nm}\right)$, DMPC $\left(L_{\mathrm{w}}=2.9 \mathrm{~nm}\right)$, and DGDG systems $\left(L_{\mathrm{w}}=2.3\right.$ $\mathrm{nm})$. The horizontal dashed lines denote the SPC/E water bulk values $\rho_{\mathrm{m}}^{\text {bulk }}=987 \mathrm{~kg} / \mathrm{m}^{3}, \varepsilon_{\text {bulk }}=70$. $(\mathrm{g})$ Interfacial water mass density profile for the graphene system (same as in $(\mathrm{d})$ ). (h) Electric field profile $E_{\perp}(z)$ in the presence of an external displacement field $D_{\perp} / \varepsilon_{0}=1.42 \mathrm{~V} / \mathrm{nm}(\mathrm{red}$ line) compared with the electric field profile $E_{\perp}^{(0)}(z)$ for $D_{\perp}=0$ (green line). The dielectric linear-response profile $\varepsilon_{\perp}{ }^{-1}$ (blue line) from polarization fluctuations is compared to the dielectric profile extracted from the induced polarizations via $\varepsilon_{\perp, \mathrm{nl}}^{-1}=\varepsilon_{0}\left(E_{\perp}-E_{\perp}^{(0)}\right) / D_{\perp}$ (black line). (i) Results for $D_{\perp} / \varepsilon_{0}=3.54 \mathrm{~V} / \mathrm{nm}$.

\section{RESULTS AND DISCUSSION}

Here we investigate the dielectric properties of water confined between planar surfaces. To span the entire range from soft to hard and from polar to nonpolar surfaces, we consider decanol (using our previously obtained results ${ }^{29}$ ) as well as graphene, phospholipid dipalmitoylphosphatidylcholine (DMPC) and glycolipid digalactosyldiacylglycerol (DGDG) bilayers; see Figure 1a. Water between graphene layers has unique frictional and chemical properties and is widely studied experimentally; ${ }^{4,6,9}$ decanol layers are prototypical self-assembled monolayers (SAMs). ${ }^{33,34}$ Phospholipid bilayers are strongly polar due to their zwitterionic nature. ${ }^{35}$ Contrarily, DGDG head groups contain multiple $\mathrm{OH}$ groups, which gives rise to a characteristic swelling behavior in water. ${ }^{36} \mathrm{We}$ find that polar surface groups significantly contribute to the dielectric response and for DMPC bilayers, but not the other polar surfaces, give rise to a giant local parallel response. From the simulated tensorial dielectric profiles we extract a tensorial dielectric slab model in terms of effective dielectric widths and effective perpendicular and parallel dielectric constants $\varepsilon_{\perp}^{\text {eff }}$ and $\varepsilon_{\|}^{\text {eff. }}$. While the effective dielectric widths differ considerably between different surface types, we find universal behavior for $\varepsilon_{\perp}^{\text {eff }}$ and $\varepsilon_{\|}^{\text {eff }}: \varepsilon_{\|}^{\text {eff }}$ stays rather constant at its bulk value down to subnanometer confinement, while $\varepsilon_{\perp}^{\text {eff }}$ decreases substantially below $1 \mathrm{~nm}$. In fact, our derived $\varepsilon_{\perp}^{\text {eff }}$ values for water between graphene agree with recent experimental results if the effective width is suitably chosen. ${ }^{30}$ Finally, our solution of the tensorial Poisson equation in planar geometry demonstrates that the electrostatic interaction between two charges with small separation depends on the product of $\varepsilon_{\perp}^{\text {eff }}$ and $\varepsilon_{\|\|}^{\text {eff }}$. Since $\varepsilon_{\perp}^{\text {eff }}$ decreases drastically for strong confinements while $\varepsilon_{\|}^{\text {eff }}$ stays approximately constant, ion interactions in strong confinement are significantly enhanced compared to bulk. For large separation, ion interactions depend on the outside medium. In particular, if the outside medium is a metal, electrostatic interactions are exponentially damped with a decay constant that depends on the ratio of $\varepsilon_{\|}^{\text {eff }}$ and $\varepsilon_{\perp}^{\text {eff }}$. These results are fundamental for the description of all electrostatics in aqueous nanoconfinement.

We simulate SPC/E water between four different planar surfaces at fixed $L$; see Figure 1a for schematics of the surface constituents. For graphene $L$ denotes the distance between the atomic centers in the graphene sheets. For the other systems (decanol, DMPC, DGDG) $L$ denotes the periodic simulation box length. Figure $1 \mathrm{~b}$ and Figure $1 \mathrm{c}$ show snapshots of the graphene and DMPC systems, respectively. The water number $N_{\text {w }}$ is fixed at a value to keep the water chemical potential or the pressure fixed. We define the water layer thickness $L_{\mathrm{w}}$ using the bulk molecular water volume $v_{\mathrm{w}}=0.0304 \mathrm{~nm}^{3}$ as $L_{\mathrm{w}}=$ $N_{\mathrm{w}} v_{\mathrm{w}} / A$, where $A$ is the lateral simulation box area. By this, $L_{\mathrm{w}}$ is equivalent to the separation between the Gibbs dividing surfaces at large surface separation (see Supporting Information sections S1 and S2 for model and simulation details).

In Figure $1 \mathrm{~d}$ we show the water mass density profiles $\rho_{\mathrm{m}}(z)$. For graphene, water exhibits pronounced layering, as expected on rigid flat surfaces. For the other softer surfaces, density profiles are smooth and change monotonically. Figure le and Figure If show the parallel $\varepsilon_{\|}(z)$ and the inverse perpendicular dielectric profiles $\varepsilon_{\perp}^{-1}(z)$ that are obtained from polarization density fluctuations at zero external field and fully account for nonlocal response effects (see Supporting Information section S3 for details). The dielectric profiles include water and surface polarizations, and the pure water contributions are shown in the Supporting Information section S3. The parallel dielectric profiles $\varepsilon_{\|}(z)$ in Figure 1e closely follow the mass density profiles for all surfaces except DMPC; for DMPC lipids we find a giant parallel response of $\varepsilon_{\|_{s}} \approx 400$ at the interface, in agreement with earlier results, ${ }^{25}$ which is due to the freely orientable zwitterionic head-group charges. The inverse perpendicular profiles $\varepsilon_{\perp}^{-1}(z)$ in Figure if look very different from $\rho_{\mathrm{m}}(z)$ and $\varepsilon_{\|}(z)$ and cross zero at least once. In fact, the divergencies of $\varepsilon_{\perp}(z)$ are unproblematic and reflect dielectric overscreening effects. ${ }^{24,37}$ 
How reliable are the linear dielectric response profiles shown in Figure 1e and Figure 1f? In Figure $1 \mathrm{~h}$ and Figure 1i we show results for graphene in the presence of external perpendicular displacement fields of $D_{\perp} / \varepsilon_{0}=1.42 \mathrm{~V} / \mathrm{nm}$ and $3.54 \mathrm{~V} / \mathrm{nm}$, respectively. Note that these fields are, due to periodic image effects, significantly larger than the nominally applied fields of $D^{\prime}{ }_{\perp} / \varepsilon_{0}=1.0 \mathrm{~V} / \mathrm{nm}$ and $2.5 \mathrm{~V} / \mathrm{nm} .{ }^{25,38}$ Green lines show the electric field $E_{\perp}^{(0)}(z)$ for $D_{\perp}=0$ and red lines show $E_{\perp}(z)$ for finite $D_{\perp}$, both obtained from integrating over the charge density profile according to $E_{\perp}(z)=\int_{-\infty}^{z} \rho\left(z^{\prime}\right) \mathrm{d} z^{\prime} / \varepsilon_{0}$. Blue lines show the linear response $\varepsilon_{\perp}^{-1}(z)$ from Figure if obtained from polarization fluctuations at zero external field, while black lines show the nonlinear dielectric profile estimated from the field-induced polarization according to $\varepsilon_{\perp, \mathrm{nl}}{ }^{-1}=\varepsilon_{0}\left(E_{\perp}-E_{\perp}^{(0)}\right) /$ $D_{\perp}$. Surprisingly, even for a relatively high applied field of $D_{\perp} /$ $\varepsilon_{0}=1.42 \mathrm{~V} / \mathrm{nm}$, which corresponds to the field created by a planar surface with charge density $\sigma=0.1 \mathrm{e} \mathrm{nm}^{-2}$, the linear dielectric profile describes the actual polarization response in Figure $1 \mathrm{~h}$ very well, meaning that our formulation of the inhomogeneous linear dielectric response is highly accurate. For $D_{\perp} / \varepsilon_{0}=3.54 \mathrm{~V} / \mathrm{nm}$ in Figure $1 \mathrm{i}$ deviations between the black and blue lines are clearly noticeable, which indicates the onset of the breakdown of linear response theory ${ }^{24,37}$ (results including higher field strengths are shown for the entire simulation box width in the Supporting Information section S3). Comparing the profiles for $\varepsilon_{\perp}^{-1}(z)$ and $E_{0}(z)$ in Figure $1 \mathrm{~h}$ and Figure 1i with $\rho_{\mathrm{m}}(z)$ in Figure $1 \mathrm{~g}$, we find all profiles to be different from each other and even the positions of the extrema not to match; we conclude that perpendicular dielectric profiles are not related to polarization and density profiles in an obvious manner.

In order to investigate the consequences of our findings for electrostatic interactions, we need to replace the complex profiles $\varepsilon_{\|}(z)$ and $\varepsilon_{\perp}^{-1}(z)$ by analytically manageable expressions, for which we choose step profiles ${ }^{29}$

$$
\varepsilon_{\alpha}^{*}(z)=\left\{\begin{array}{cl}
\varepsilon_{\alpha}^{\text {eff }} & \text { if }|z| \leq L_{\alpha}^{\text {eff }} / 2 \\
1 & \text { if }|z|>L_{\alpha}^{\text {eff }} / 2
\end{array}\right.
$$

The parameters, i.e., the effective dielectric constants $\varepsilon_{\alpha}^{\text {eff }}$ and the effective dielectric widths $L_{\alpha}^{\text {eff }}$ for parallel and perpendicular directions $\alpha=\perp, \|$, are obtained using dielectric effective medium theory in the following manner: For the perpendicular component we demand that the integral over $E_{\perp}(z)$ induced by a constant $D_{\perp}$ field is exactly reproduced by the box profile, which leads to ${ }^{29}$

$$
\frac{1}{\varepsilon_{\perp}^{\text {eff }}}=1+\frac{\int_{-L / 2}^{L / 2} \varepsilon_{\perp}^{-1}(z) \mathrm{d} z-L}{L_{\perp}^{\text {eff }}}
$$

For the parallel component we demand that the integral over $D_{\|}(z)$ induced by a constant $E_{\|}$field is reproduced by the box profile, which leads to

$$
\varepsilon_{\|}^{\text {eff }}=1+\frac{\int_{-L / 2}^{L / 2} \varepsilon_{\|}(z) \mathrm{d} z-L}{L_{\|}^{\text {eff }}}
$$

See Supporting Information section S3 for detailed derivations. Obviously, the four parameters $\varepsilon_{\alpha}^{\text {eff }}$ and $L_{\alpha}^{\text {eff }}$ of the effective model cannot be uniquely derived from the simulated dielectric profiles using the two eqs 2 and 3. However, we expect that for large water slab thickness $L_{\mathrm{w}}$ the effective dielectric constants $\varepsilon_{\alpha}^{\text {eff }}$ approach the bulk value $\varepsilon_{\text {bulk; }}$ this asymptotic property can be used to reduce the number of free parameters. In Figure 2 we show $L_{\alpha}^{\text {eff }}-L_{\mathrm{w}}$, determined from

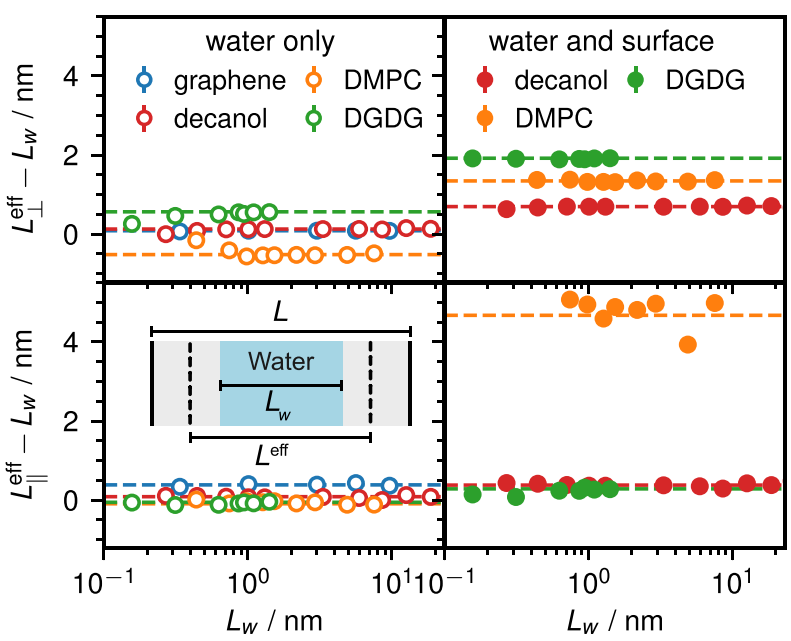

Figure 2. Difference between dielectric width and water slab thickness, $L_{\perp}^{\text {eff }}-L_{\mathrm{w}}$ (top) and $L_{\|}^{\text {eff }}-L_{\mathrm{w}}$ (bottom), according to eqs 2 and 3 , using the SPC/E value $\varepsilon_{\alpha}^{\text {eff }}=\varepsilon_{\text {bulk }}=70$. Horizontal dashed lines denote the asymptotic values $\bar{L}_{\perp}^{\text {eff }}-L_{\mathrm{w}}$ and $\bar{L}_{\|}^{\text {eff }}-L_{\mathrm{w}}$, obtained by averaging the data for $L_{\mathrm{w}}>1 \mathrm{~nm}$. The left panels show the water contribution only, and the right panels include the surface polarization contribution.

eqs 2 and 3, as a function of $L_{\mathrm{w}}$ for fixed $\varepsilon_{\alpha}^{\text {eff }}=\varepsilon_{\text {bulk }}=70 .{ }^{39}$ The top row shows results for the perpendicular component, the bottom row for the parallel component. In the left panels, we only use the water polarization contribution. In the right panels we use the full polarization including the surface contribution. The differences between the left and right panels indicate the surface contribution to the dielectric response, which is particularly large for the parallel DMPC component (note that there is no surface contribution for our graphene model, and therefore the graphene data are not included in the right figures). Generally, $L_{\alpha}^{\text {eff }}-L_{\mathrm{w}}$ including the surface polarization contribution is positive, meaning that aqueous interfaces exhibit a positive excess dielectric contribution. As expected, we find that $L_{\alpha}^{\text {eff }}-L_{\mathrm{w}}$ becomes constant for $L_{\mathrm{w}}>1 \mathrm{~nm}$. We thus obtain asymptotic estimates $\bar{L}_{\alpha}^{\text {eff }}-L_{\mathrm{w}}$ by averaging the data for $L_{\mathrm{w}}>1 \mathrm{~nm}$, indicated by broken horizontal lines.

Fixing the effective dielectric constants inside the water slab and determining the effective dielectric widths from simulations, as done in Figure 2, constitute one choice of an effective dielectric model. We now explore an alternative model, where we do not fix the effective dielectric constants. In this case we can derive effective dielectric constants $\varepsilon_{\alpha}^{\text {eff }}$ in a unique fashion by using the asymptotic effective dielectric widths $\bar{L}_{\alpha}^{\text {eff }}$ determined in Figure 2 . We do this first for graphene because here experimental data exist that we can compare with. Figure 3 a shows $\varepsilon_{\perp}^{\text {eff }}$ according to eq 2 using our graphene simulation data as a function of the graphene layer distance $L$ for different choices of $L_{\perp}^{\text {eff }}$ (blue spheres). We see that $\varepsilon_{\perp}^{\text {eff }}$ decreases significantly with rising $L_{\perp}^{\text {eff }}$, which demonstrates a strong dependence of the effective dielectric constants on the effective dielectric width used. For the asymptotic value $L_{\perp}^{\text {eff }}=\bar{L}_{\perp}^{\text {eff }}=L-0.3 \mathrm{~nm}$, obtained from $\bar{L}_{\perp}^{\text {eff }}-$ $L_{\mathrm{w}}=0.08$ from Figure 2 and $L-L_{\mathrm{w}}=0.38$ from Supporting 

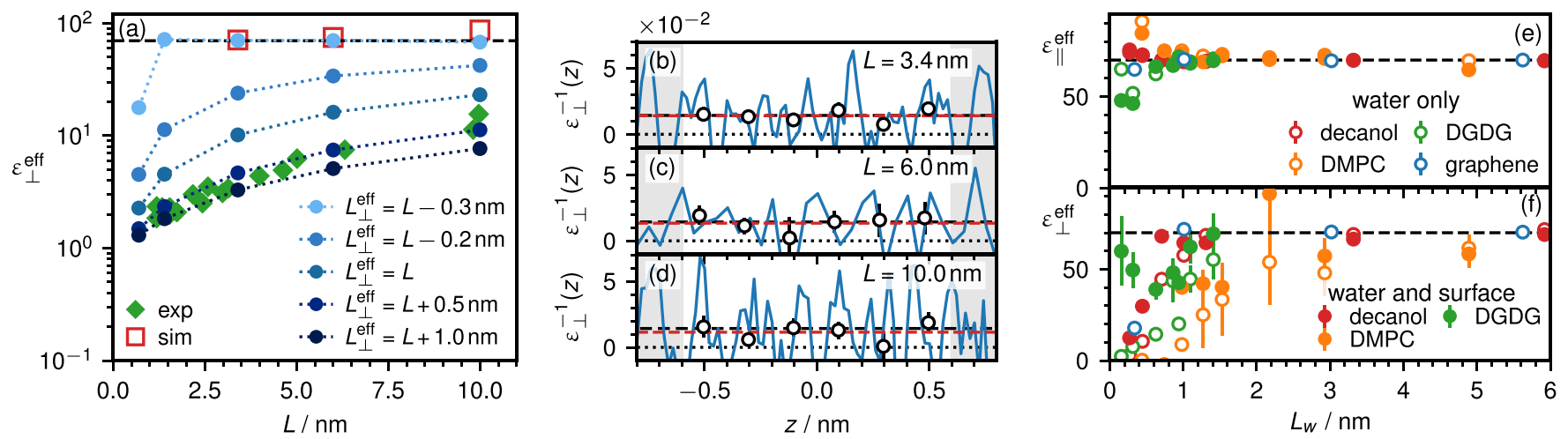

Figure 3. (a) Perpendicular effective dielectric constant $\varepsilon_{\perp}^{\text {eff }}$ from simulations according to eq 2 for water between graphene sheets as a function of the graphene separation $L$ for different values of the effective dielectric width $L_{\perp}^{\text {eff }}$ (blue symbols). Open red squares show the dielectric constant directly obtained from averages over the inner region of the graphene system in (b) $-(d)$. Green diamonds show the perpendicular effective dielectric constant from experiments with water between graphene and boron nitride layers using the experimentally employed conversion $L=h+$ $0.34 \mathrm{~nm}$, where $h$ is the reported water slab thickness. ${ }^{30}(\mathrm{~b}-\mathrm{d})$ Inverse dielectric profiles $\varepsilon_{\perp}{ }^{-1}(z)$ from graphene simulations in the slab center for graphene separations $L=3.4,6.0,10.0 \mathrm{~nm}$ (blue lines). Circles show averages over bins of thickness $0.2 \mathrm{~nm}$, and horizontal red dashed lines denote the average over the central region of thickness $1.2 \mathrm{~nm}$ and are in very good agreement with the SPC/E bulk value of $1 / \varepsilon_{\text {bulk }}=1 / 70$, shown as horizontal black dashed lines. The horizontal black dotted lines denote zero. (e) Parallel effective dielectric constants for all simulated systems according to eq 2 using $\bar{L}_{\|}^{\text {eff }}$ from Figure 2. Open symbols denote the water-only contribution, and full symbols include the surface polarization contribution. (f) Perpendicular effective dielectric constants using $\bar{L}_{\perp}^{\text {eff }}$ from Figure 2.

Information section $\mathrm{S} 2$, we see that the effective dielectric constant stays at its bulk value down to $L=1.4 \mathrm{~nm}$. For the slightly larger value $L_{\perp}^{\text {eff }}=L$, where the effective dielectric width equals the graphene separation, $\varepsilon_{\perp}^{\text {eff }}$ is significantly reduced over the entire $L$ range studied. The experimental $\varepsilon_{\perp}^{\text {eff }}$ for water confined between planar boron nitride and graphene layers, green diamonds, has been obtained by choosing $L_{\perp}^{\text {eff }}=L-0.34$ $\mathrm{nm}$ in the extraction from the experimental data. ${ }^{30}$ It is seen to compare well with our simulations for $L_{\perp}^{\text {eff }}=L+0.5 \mathrm{~nm}$. This shift of $L_{\perp}^{\text {eff }}$ by $0.84 \mathrm{~nm}$ from the scenario where the effective dielectric constant stays at its bulk value down to graphene separation of $1 \mathrm{~nm}$ has been interpreted in terms of water interfacial layers with a much reduced dielectric constant ${ }^{30}$ (see Supporting Information section S4 for details). It is not clear why these interfacial layers are apparently absent in simulations, which follows from the fact that the simulation data for $L_{\perp}^{\text {eff }}=\bar{L}_{\perp}^{\text {eff }}=L-0.3 \mathrm{~nm}$ differ substantially from the experimental data. One possible explanation could be that the usage of a nonpolarizable force field for the graphene layers produces an unrealistically high water density. However, Figure 2 suggests that surface polarizability increases the interfacial dielectric response and thus would further increase deviations from experiment. In fact, we will next demonstrate how simulations allow unambiguous determination of the correct value of the effective dielectric widths to be used in the extraction of effective dielectric constants.

The simulated values of $\varepsilon_{\perp}^{\text {eff }}$ in Figure 3 a are consistent with experiments only for a specific choice of $L_{\perp}^{\text {eff }}$, which significantly differs from the value used in the analysis of the experimental data. This prompts the question of what the actual values of $\varepsilon_{\perp}^{\text {eff }}$ and $\varepsilon_{\|}^{\text {eff }}$ are that should be used in coarse-grained models for confined water slabs. Simulations provide the answer since they can look into the water slab and determine the local dielectric constant, which is model-independent: In Figure $3 \mathrm{~b}-\mathrm{d}$ we show the strongly fluctuating $\varepsilon_{\perp}^{-1}(z)$ profiles in the central region between graphene at three different separations (blue lines). We also show averages over bins of width $0.2 \mathrm{~nm}$ (open spheres) and averages over the inner region of thickness $1.2 \mathrm{~nm}$ (red dashed lines), which agree accurately with the
SPC $/ E$ bulk value $1 / \varepsilon_{\text {bulk }}=0.014$ (black dashed lines). The averages over the inner regions are included in Figure 3a as red squares and agree perfectly with the prediction using the asymptotic dielectric width $L_{\perp}^{\text {eff }}=\bar{L}_{\perp}^{\text {eff }}=L-0.3 \mathrm{~nm}$. We conclude that only the usage of the asymptotic effective dielectric width $\bar{L}_{\perp}^{\text {eff }}$ leads to physically sound estimates of effective dielectric constants $\varepsilon_{\perp}^{\text {eff }}$ and $\varepsilon_{\|}^{\text {eff }}$ that agree with the local dielectric constants in the water slab center. Coming back to the disagreement between the simulation and experimental results in Figure 3a, we mention that this could be explained either by the presence of less water than assumed in the experiments, for example, due to graphene deformation or slow water filling kinetics, or by more water present in the simulations compared to the experiment, for example, due to force field issues.

In Figure $3 \mathrm{e}$ and Figure $3 \mathrm{f}$ we show the effective dielectric constants $\varepsilon_{\|}^{\text {eff }}$ and $\varepsilon_{\perp}^{\text {eff }}$ for all four simulated systems using $\bar{L}_{\|}^{\text {eff }}$ and $\bar{L}_{\perp}^{\text {eff }}$ from Figure 2 . We find very little difference between the results excluding (open circles) and including the surface polarization (full circles). The effective parallel dielectric constant $\varepsilon_{\|}^{\text {eff }}$ is rather independent of $L_{w}$, while the perpendicular component $\varepsilon_{\perp}^{\text {eff }}$ decreases significantly below $L_{\mathrm{w}}$ $\approx 1-2 \mathrm{~nm}$, which has been shown to be due to anticorrelated water dipoles. ${ }^{29}$ We conclude that dielectric properties of water are rather independent of the confining medium if the dielectric widths $L_{\|}^{\text {eff }}$ and $L_{\perp}^{\text {eff }}$ are properly accounted for. As mentioned before, alternatively, one could define an effective model using the bulk dielectric constant of water and employing water-slab-thickness-dependent dielectric widths $\bar{L}_{\|}^{\text {eff }}$ and $\bar{L}_{\perp}^{\text {eff }}$. So there are different effective dielectric models that describe the same physics.

We now derive effective electrostatic interactions for pointlike charges embedded in a linear anisotropic dielectric medium using the effective dielectric constants extracted from simulations. The electrostatic potential $\mathcal{G}\left(\mathbf{r}, \mathbf{r}^{\prime}\right)$ created at $\mathbf{r}$ by a unit charge at position $\mathbf{r}^{\prime}$ follows from Poisson's equation

$$
\varepsilon_{0}\left\{\nabla\left[\boldsymbol{\varepsilon}(\mathbf{r}) \cdot \nabla \mathcal{G}\left(\mathbf{r}, \mathbf{r}^{\prime}\right)\right]\right\}=-\delta\left(\mathbf{r}-\mathbf{r}^{\prime}\right)
$$


We assume the diagonal dielectric tensor $\boldsymbol{\varepsilon}(\mathbf{r})$ to be piecewise constant in three spatial regions, as schematically shown in Figure 4. In the Supporting Information section S5 we show
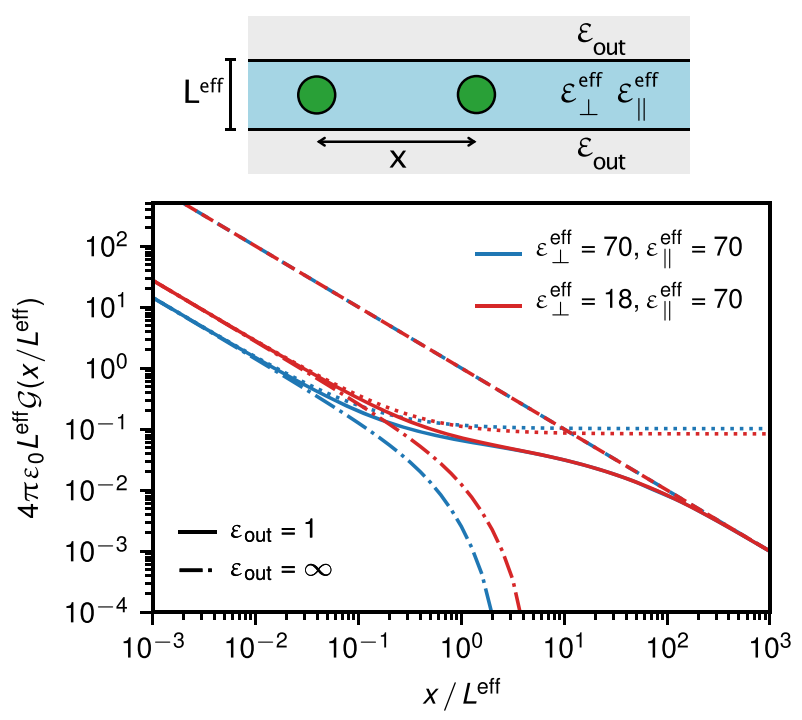

Figure 4. Rescaled electrostatic potential $\mathcal{G}\left(x / L^{\text {eff }}\right)$ for two charges at a distance $x$ in the water slab center. Solid lines show the exact solution, dotted lines the asymptotic result of eq 5 valid for $x / L^{\text {eff }} \rightarrow$ 0 , dashed lines the asymptotic result of eq 6 valid for $x / L^{\text {eff }} \rightarrow \infty$ for a confining vacuum medium $\left(\varepsilon_{\text {out }}=1\right)$. Dashed-dotted lines show the exact solution for a confining metallic medium with $\varepsilon_{\text {out }} \rightarrow \infty$. All results are presented for two different slab dielectric constants.

that a more detailed model with five regions that accounts for the different effective dielectric widths $L_{\|}^{\text {eff }}$ and $L_{\perp}^{\text {eff }}$ gives very similar results; we therefore use here $L^{\text {eff }} \equiv L_{\|}^{\text {eff }}=L_{\perp}^{\text {eff }}$. In Figure 4 we show $G\left(x / L^{\text {eff }}\right)$ as a function of the distance $x / L^{\text {eff }}$ between two charges positioned in the water slab center. The solid and dashed-dotted lines show results if the outer medium is vacuum $\left(\varepsilon_{\text {out }}=1\right)$ and metallic $\left(\varepsilon_{\text {out }} \rightarrow \infty\right)$, respectively. Red lines show results for $\varepsilon_{\|}^{\text {eff }}=70$ and $\varepsilon_{\perp}^{\text {eff }}=18$, representing water between graphene layers at a separation $L=0.7 \mathrm{~nm}$ (see Figure $3 \mathrm{e}$ and Figure $3 \mathrm{f}$ ), while blue lines are results for $\varepsilon_{\|}^{\text {eff }}=\varepsilon_{\perp}^{\text {eff }}=70$, relevant for water between graphene layers that are $L=1.4 \mathrm{~nm}$ or farther apart (the derivation is detailed in the Supporting Information section S5). The reduction of $\varepsilon_{\perp}^{\text {eff }}$ significantly enhances electrostatic interactions at low separation, while metallic confinement reduces their range. The dotted lines in Figure 4 show the limiting results for small $x$,

$$
\lim _{x \rightarrow 0} \mathcal{G}(x) \simeq \frac{1}{4 \pi \varepsilon_{0} \sqrt{\varepsilon_{\perp} \varepsilon_{\|}}|x|}
$$

and the broken lines show the limiting results for large $x$ and outer vacuum medium with $\varepsilon_{\text {out }}=1$,

$$
\lim _{x \rightarrow \infty} \mathcal{G}(x) \simeq \frac{1}{4 \pi \varepsilon_{0} \varepsilon_{\text {out }}|x|}
$$

Interestingly, eq 5 , which describes the interaction accurately for distances below $x / L^{\text {eff }}=0.5$, depends inversely on the product $\sqrt{\varepsilon_{\|} \varepsilon_{\perp}}$, which explains why interactions increase at strong confinement where $\varepsilon_{\perp}$ decreases significantly. For large separations $x / L^{\text {eff }}>1$ the slab dielectric constants become irrelevant and a slow crossover to eq 6 is observed (in the
Supporting Information section S5 a crossover formula is provided, helpful for future coarse-grained simulations). The asymptotic large-distance interaction in metallic confinement with $\varepsilon_{\text {out }} \rightarrow \infty$ reads

$$
\lim _{x \rightarrow \infty} \mathcal{G}(x) \simeq \frac{1}{4 \pi \varepsilon_{0} \varepsilon_{\|}^{3 / 4} \varepsilon_{\perp}^{1 / 4}} \sqrt{\frac{8}{x / L}} e^{-\pi \sqrt{\frac{\varepsilon_{\perp}}{\varepsilon_{\|}}} x / L}
$$

and exhibits an exponential decay with a universal decay constant that depends on the ratio $\varepsilon_{\|}^{\text {eff }} / \varepsilon_{\perp}^{\text {eff }}$, which explains the results in Figure 4 (dashed-dotted lines); see Supporting Information section S5 for derivations of all formulas.

\section{CONCLUSION}

We extract dielectric tensorial profiles of confined water from atomistic simulations of four fundamentally different planar systems. Using effective medium theory, we convert dielectric profiles into asymptotic dielectric slab widths and water-slabthickness dependent effective dielectric constants. Recent experimental measurements of the effective perpendicular dielectric constant of water between graphene and boronnitride layers ${ }^{30}$ can be reproduced if the effective dielectric width used in the extraction is suitably adjusted. Regardless of the system, by choice of values for the effective dielectric widths that follow from our asymptotic analysis for large water slab thicknesses, the parallel effective dielectric constants stay close to the bulk value down to the smallest confinement while the perpendicular effective dielectric constants significantly decrease for confinement below a nanometer. This finding is confirmed by comparison with the local dieletric constant determined in the slab center. An exact solution of the Poisson equation in anisotropic dielectric slabs demonstrates that electrostatic interactions between charges at small separation depend on the product of $\varepsilon_{\|}^{\text {eff }}$ and $\varepsilon_{\perp}^{\text {eff }}$. If the confining medium is metallic, the charge-charge interactions for large distances decay exponentially with a decay constant that depends on the

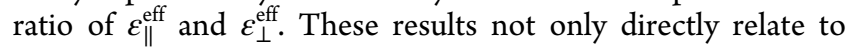
current experiments but also provide the framework for future coarse-grained simulations and theories for electrostatics in aqueous confinement.

\section{ASSOCIATED CONTENT}

\section{SI Supporting Information}

The Supporting Information is available free of charge at https://pubs.acs.org/doi/10.1021/acs.jpcb.0c01967.

Simulation details, determination of the effective water slab thickness for graphene system, derivation of the equations for dielectric profiles and dielectric box model, dielectric constant of an effective capacitor model, derivation of the tensorial electrostatic model including limiting cases and a heuristic formula, and correlation time of the total dipole moment in confinement (PDF)

\section{AUTHOR INFORMATION}

\section{Corresponding Author}

Roland R. Netz - Fachbereich Physik, Freie Universität Berlin, 14195 Berlin, Germany; Email: rnetz@physik.fu-berlin.de

\section{Authors}

Philip Loche - Fachbereich Physik, Freie Universität Berlin, 14195 Berlin, Germany; (1) orcid.org/0000-0002-9112-0010

Cihan Ayaz - Fachbereich Physik, Freie Universität Berlin, 14195 Berlin, Germany 
Amanuel Wolde-Kidan - Fachbereich Physik, Freie Universität Berlin, 14195 Berlin, Germany

Alexander Schlaich - Université Grenoble Alpes, CNRS, LIPhy,

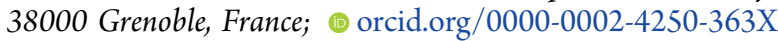

Complete contact information is available at:

https://pubs.acs.org/10.1021/acs.jpcb.0c01967

\section{Notes}

The authors declare no competing financial interest.

\section{ACKNOWLEDGMENTS}

The authors acknowledge the Max-Planck Water Initiative for funding and the North-German Supercomputing Alliance (HLRN) for providing HPC resources that have contributed to the research results reported in this paper.

\section{REFERENCES}

(1) Schoch, R. B.; Han, J.; Renaud, P. Transport phenomena in nanofluidics. Rev. Mod. Phys. 2008, 80, 839-883.

(2) Bocquet, L.; Charlaix, E. Nanofluidics, from bulk to interfaces. Chem. Soc. Rev. 2010, 39, 1073-1095.

(3) Geim, A. K.; Grigorieva, I. V. Van der Waals heterostructures. Nature 2013, 499, 419-425.

(4) Radha, B.; Esfandiar, A.; Wang, F. C.; Rooney, A. P.; Gopinadhan, K.; Keerthi, A.; Mishchenko, A.; Janardanan, A.; Blake, P.; Fumagalli, L.; et al. Molecular transport through capillaries made with atomic-scale precision. Nature 2016, 538, 222-225.

(5) Gravelle, S.; Yoshida, H.; Joly, L.; Ybert, C.; Bocquet, L. Carbon membranes for efficient water-ethanol separation. J. Chem. Phys. 2016, 145, 124708.

(6) Hong, S.; Constans, C.; Surmani Martins, M. V.; Seow, Y. C.; Guevara Carrió, J. A.; Garaj, S. Scalable Graphene-Based Membranes for Ionic Sieving with Ultrahigh Charge Selectivity. Nano Lett. 2017, 17, 728-732.

(7) Zhou, K.-G.; Vasu, K. S.; Cherian, C. T.; Neek-Amal, M.; Zhang, J. C.; Ghorbanfekr-Kalashami, H.; Huang, K.; Marshall, O. P.; Kravets, V. G.; Abraham, J.; et al. Electrically controlled water permeation through graphene oxide membranes. Nature 2018, 559, 236-240.

(8) Simoncelli, M.; Ganfoud, N.; Sene, A.; Haefele, M.; Daffos, B.; Taberna, P.-L.; Salanne, M.; Simon, P.; Rotenberg, B. Blue Energy and Desalination with Nanoporous Carbon Electrodes: Capacitance from Molecular Simulations to Continuous Models. Phys. Rev. X 2018, 8, 021024.

(9) Mouterde, T.; Keerthi, A.; Poggioli, A. R.; Dar, S. A.; Siria, A.; Geim, A. K.; Bocquet, L.; Radha, B. Molecular streaming and its voltage control in ångström-scale channels. Nature 2019, 567, 87-90.

(10) Gopinadhan, K.; Hu, S.; Esfandiar, A.; Lozada-Hidalgo, M.; Wang, F. C.; Yang, Q.; Tyurnina, A. V.; Keerthi, A.; Radha, B.; Geim, A. K. Complete steric exclusion of ions and proton transport through confined monolayer water. Science 2019, 363, 145-148.

(11) Leikin, S.; Parsegian, V. A.; Rau, D. C.; Rand, R. P. Hydration Forces. Annu. Rev. Phys. Chem. 1993, 44, 369-395.

(12) Du, Q.; Freysz, E.; Shen, Y. R. Vibrational spectra of water molecules at quartz/water interfaces. Phys. Rev. Lett. 1994, 72, 238241.

(13) Maggs, A. C.; Everaers, R. Simulating Nanoscale Dielectric Response. Phys. Rev. Lett. 2006, 96, 230603.

(14) Li, T.-D.; Gao, J.; Szoszkiewicz, R.; Landman, U.; Riedo, E. Structured and viscous water in subnanometer gaps. Phys. Rev. B: Condens. Matter Mater. Phys. 2007, 75, 115415.

(15) Snyder, P. W.; Lockett, M. R.; Moustakas, D. T.; Whitesides, G. $\mathrm{M}$. Is it the shape of the cavity, or the shape of the water in the cavity? Eur. Phys. J.: Spec. Top. 2014, 223, 853-891.

(16) O’Hern, S. C.; Boutilier, M. S. H.; Idrobo, J.-C.; Song, Y.; Kong, J.; Laoui, T.; Atieh, M.; Karnik, R. Selective Ionic Transport through
Tunable Subnanometer Pores in Single-Layer Graphene Membranes. Nano Lett. 2014, 14, 1234-1241.

(17) Jain, T.; Rasera, B. C.; Guerrero, R. J. S.; Boutilier, M. S. H.; O’Hern, S. C.; Idrobo, J.-C.; Karnik, R. Heterogeneous subcontinuum ionic transport in statistically isolated graphene nanopores. Nat. Nanotechnol. 2015, 10, 1053-1057.

(18) Muñoz-Santiburcio, D.; Marx, D. Chemistry in nanoconfined water. Chem. Sci. 2017, 8, 3444-3452.

(19) Chakraborty, S.; Kumar, H.; Dasgupta, C.; Maiti, P. K. Confined Water: Structure, Dynamics, and Thermodynamics. Acc. Chem. Res. 2017, 50, 2139-2146.

(20) Ruiz-Barragan, S.; Muñoz-Santiburcio, D.; Marx, D. Nanoconfined Water within Graphene Slit Pores Adopts Distinct Confinement-Dependent Regimes. J. Phys. Chem. Lett. 2019, 10, 329-334.

(21) Ling, X.; Bonn, M.; Domke, K. F.; Parekh, S. H. Correlated interfacial water transport and proton conductivity in perfluorosulfonic acid membranes. Proc. Natl. Acad. Sci. U. S. A. 2019, 116, 87158720.

(22) Honig, B.; Nicholls, A. Classical electrostatics in biology and chemistry. Science 1995, 268, 1144-1149.

(23) Ben-Yaakov, D.; Andelman, D.; Podgornik, R. Dielectric decrement as a source of ion-specific effects. J. Chem. Phys. 2011, 134, 074705 .

(24) Bonthuis, D. J.; Gekle, S.; Netz, R. R. Profile of the static permittivity tensor of water at interfaces: consequences for capacitance, hydration interaction and ion adsorption. Langmuir 2012, 28, 7679-7694.

(25) Stern, H. A.; Feller, S. E. Calculation of the dielectric permittivity profile for a nonuniform system: Application to a lipid bilayer simulation. J. Chem. Phys. 2003, 118, 3401-3412.

(26) Ballenegger, V.; Hansen, J.-P. Dielectric permittivity profiles of confined polar fluids. J. Chem. Phys. 2005, 122, 114711.

(27) Cui, H.-B.; Takahashi, K.; Okano, Y.; Kobayashi, H.; Wang, Z.; Kobayashi, A. Dielectric Properties of Porous Molecular Crystals That Contain Polar Molecules. Angew. Chem., Int. Ed. 2005, 44, 65086512.

(28) Zhang, C.; Gygi, F.; Galli, G. Strongly Anisotropic Dielectric Relaxation of Water at the Nanoscale. J. Phys. Chem. Lett. 2013, 4, $2477-2481$.

(29) Schlaich, A.; Knapp, E. W.; Netz, R. R. Water Dielectric Effects in Planar Confinement. Phys. Rev. Lett. 2016, 117, 048001.

(30) Fumagalli, L.; Esfandiar, A.; Fabregas, R.; Hu, S.; Ares, P.; Janardanan, A.; Yang, Q.; Radha, B.; Taniguchi, T.; Watanabe, K.; et al. Anomalously low dielectric constant of confined water. Science 2018, 360, 1339-1342.

(31) Ruiz-Barragan, S.; Muñoz-Santiburcio, D.; Körning, S.; Marx, D. Quantifying anisotropic dielectric response properties of nanoconfined water within graphene slit pores. Phys. Chem. Chem. Phys. 2020, DOI: 10.1039/D0CP00916D.

(32) Zhang, C. Note: On the dielectric constant of nanoconfined water. J. Chem. Phys. 2018, 148, 156101.

(33) Hillier, A. C.; Kim, S.; Bard, A. J. Measurement of DoubleLayer Forces at the Electrode/Electrolyte Interface Using the Atomic Force Microscope: Potential and Anion Dependent Interactions. J. Phys. Chem. 1996, 100, 18808-18817.

(34) Rentsch, S.; Siegenthaler, H.; Papastavrou, G. Diffuse Layer Properties of Thiol-Modified Gold Electrodes Probed by Direct Force Measurements. Langmuir 2007, 23, 9083-9091.

(35) Kanduč, M.; Schneck, E.; Netz, R. R. Hydration Interaction between Phospholipid Membranes: Insight into Different Measurement Ensembles from Atomistic Molecular Dynamics Simulations. Langmuir 2013, 29, 9126-9137.

(36) Kanduč, M.; Schlaich, A.; de Vries, A. H.; Jouhet, J.; Maréchal, E.; Demé, B.; Netz, R. R.; Schneck, E. Tight cohesion between glycolipid membranes results from balanced water-headgroup interactions. Nat. Commun. 2017, 8, 14899.

(37) Kornyshev, A. A.; Sutmann, G. Nonlocal Dielectric Saturation in Liquid Water. Phys. Rev. Lett. 1997, 79, 3435-3438. 
(38) Loche, P.; Wolde-Kidan, A.; Schlaich, A.; Bonthuis, D. J.; Netz, R. R. Comment on "Hydrophobic Surface Enhances Electrostatic Interaction in Water. Phys. Rev. Lett. 2019, 123, 049601.

(39) Vega, C.; Abascal, J. L. F. Simulating water with rigid nonpolarizable models: a general perspective. Phys. Chem. Chem. Phys. 2011, 13, 19663-19688. 